\section{Descontinuidades contraceptivas no uso do contraceptivo hormonal oral, injetável e do preservativo masculino}

\section{Contraceptive discontinuities in the use of oral and injectable hormonal contraceptives, and male condoms}

\section{Discontinuidades contraceptivas en el uso del anticonceptivo hormonal oral o inyectable y del preservativo masculino}

\section{Resumo}

O objetivo foi estimar as taxas de descontinuidade total no uso do contraceptivo hormonal oral, injetável e do preservativo masculino, bem como verificar as taxas de interrupção por abandono e por troca para método mais eficaze menos eficaz. Dados de 2.051 mulheres usuárias de unidades básicas de saúde de três capitais brasileiras foram coletados por meio do calendário contraceptivo. Os resultados mostraram que 24,5\% das usuárias do contraceptivo hormonal oral, 33,5\% das usuárias de contraceptivo hormonal injetável e 39\% das usuárias do preservativo masculino haviam descontinuado o uso do método até 12 meses de uso, independentemente da razão. Houve pouca variação nas taxas entre capitais, mas não no método utilizado. A principal razão para descontinuar o uso do método contraceptivo foi por querer engravidar (20,8\%). Um total de $20 \%$ das mulheres engravidou enquanto usava algum método, e essa proporção alcançou $25,7 \%$ entre usuárias do preservativo masculino. Ressalta-se que, após 12 meses de uso, a taxa de abandono por razões relacionadas ao método contraceptivo foi de 11,4\% entre usuárias do injetável. A taxa de troca para método mais eficaz foi de 15,9\% entre usuárias do preservativo masculino, e a taxa de troca para método menos eficaz foi de 16,3\% entre usuárias do contraceptivo hormonal injetável. As taxas de descontinuidade contraceptiva foram altas e variaram conforme o tipo de método contraceptivo utilizado.

Contracepção; Anticoncepcionais Orais; Contraceptivos Hormonais;

Preservativos; Saúde Sexual e Reprodutiva

\author{
Ana Luiza Vilela Borges 1 \\ Christiane Borges do Nascimento Chofakian ${ }^{2}$ \\ Osmara Alves Viana 3 \\ Eveline do Amor Divino 4
}

doi: 10.1590/0102-311X00014220

\author{
Correspondência \\ A. L. V. Borges \\ Escola de Enfermagem, Universidade de São Paulo. \\ Rua Dr. Enéias de Carvalho Aguiar 419, São Paulo, SP \\ 05403-000, Brasil. \\ alvilela@usp.br \\ 1 Escola de Enfermagem, Universidade de São Paulo, São Paulo, \\ Brasil. \\ 2 Escola de Ciências, Artes e Humanidades, Universidade de São \\ Paulo, São Paulo, Brasil. \\ 3 Faculdade de Medicina, Universidade de São Paulo, São Paulo \\ Brasil \\ 4 Faculdade de Enfermagem, Universidade Federal do Mato \\ Grosso, Cuiabá, Brasil.
}




\section{Introdução}

No Brasil, a última Pesquisa Nacional de Demografia e Saúde (PNDS) de 2006 mostrou que a prevalência de uso de métodos contraceptivos entre as mulheres brasileiras era de 67,8\% 1. Embora relevante para avaliar as políticas e os programas na área de saúde reprodutiva, tal indicador não considera a dinâmica contraceptiva, ou seja, a duração de uso do método, as interrupções ou as mudanças no uso. Esse dinamismo pode ser averiguado por estimativa da taxa de descontinuidade contraceptiva, que é composta por abandono (quando a mulher interrompe o uso do método contraceptivo e não adota outro imediatamente), por troca (quando a mulher muda o método contraceptivo para um mais eficaz ou para um menos eficaz) e por falha (quando a mulher engravida usando o método contraceptivo) 2,3.

As descontinuidades no uso de métodos contraceptivos por razões que não sejam para engravidar contribuem para a ocorrência de gravidezes não intencionais 4 , relatadas por $55,4 \%$ das puérperas do país 5 , assim como para a ocorrência de abortamentos induzidos 6,7 , que resultam em sérias implicações na morbimortalidade materna, por sua restrição legal no Brasil. Portanto, estudos têm procurado mensurar o quanto mulheres que usam métodos contraceptivos interrompem seu uso, a despeito de não quererem engravidar, e/ou alternam os tipos de métodos, nem sempre com opção por um método mais eficaz. Tal mensuração pode servir como base para avaliação indireta da qualidade da atenção em contracepção e até mesmo da própria satisfação das usuárias em relação ao método contraceptivo em uso ${ }^{8}$.

Diversos estudos mostraram que a ocorrência de descontinuidade contraceptiva é comum 3,9,10. Estima-se que um terço das mulheres que iniciam o uso de método vai descontinuá-lo dentro de um ano ${ }^{3}$, sendo o principal determinante o tipo de método utilizado. Os métodos que não requerem a ação de um profissional de saúde para interromper o uso são os que apresentam maiores taxas de descontinuidade, como o contraceptivo hormonal oral ou o preservativo masculino 8,11,12,13.

Apesar de inúmeros estudos sobre as descontinuidades contraceptivas terem sido conduzidos em nível internacional, principalmente com dados oriundos de inquéritos do tipo Demographic and Health Survey (DHS), os estudos conduzidos com mulheres brasileiras são ainda escassos. Além disso, são baseados em dados coletados há mais de 20 anos 8,14 ou consideraram apenas o uso do contraceptivo oral 13,15. Dessa forma, estudos que explorem os padrões de uso de método contraceptivo entre brasileiras são necessários, pois a compreensão da dinâmica contraceptiva é crucial para a elaboração, implementação e avaliação de ações de atenção em contracepção. O presente estudo tem como objetivo estimar as taxas de descontinuidade contraceptiva no uso dos métodos contraceptivos reversíveis mais utilizados no Brasil (contraceptivo hormonal oral ou injetável e preservativo masculino), nos 12 meses posteriores ao início de uso do método, entre mulheres usuárias de unidades básicas de saúde (UBS), além das taxas específicas de descontinuidade por abandono e por troca para método mais ou menos eficaz.

\section{Métodos}

Trata-se de estudo quantitativo, do tipo transversal, conduzido com mulheres de 18 a 49 anos de idade, usuárias de UBS das cidades de São Paulo (São Paulo), Aracaju (Sergipe) e Cuiabá (Mato Grosso), Brasil, entre 2015 e 2017.

O número de mulheres entrevistadas foi baseado nos seguintes parâmetros: proporção de mulheres que usava método contraceptivo 1, nível de 95\% de confiança, erro de amostragem de 5\% e efeito do delineamento (deff) igual a 2 ( $\mathrm{n}=768$ mulheres em São Paulo e n = 385, em Aracaju e em Cuiabá). Foram acrescidos os percentuais de $25 \%$ de recusa ou perda e de $33 \%$ que não seriam elegíveis para a entrevista devido aos critérios de exclusão, que foram nunca ter tido relação sexual e ter feito laqueadura ou ter parceiro vasectomizado há mais de cinco anos, o que resultou na necessidade de se obter, ao menos, 1.000 entrevistas válidas em São Paulo e 482 em Aracaju e em Cuiabá. O plano amostral foi conduzido por meio de amostragem por conglomerados em dois estágios. A unidade de primeiro estágio foram as UBS, sorteadas com probabilidade proporcional ao tamanho, medido pelo número de exames citopatológicos cervicovaginais realizados em 2014. Por esse critério, foram sorteadas 38 UBS em São Paulo, dentre 441; 19 em Aracaju, dentre 43; e 19 em Cuiabá, dentre 93. Nas três capitais, 
a pesquisa foi realizada em três dias consecutivos em cada UBS e, em cada dia, foram entrevistadas nove mulheres, totalizando 27 entrevistas válidas por UBS. As UBS sorteadas estão geograficamente distribuídas por todas as regiões dessas cidades, incluindo região central e extremos da periferia. No segundo estágio de seleção, foram selecionadas as mulheres que seriam entrevistadas em cada UBS, segundo os seguintes critérios: (1) mulheres que aguardavam atendimento para realização do exame citopatológico cervicovaginal; (2) mulheres que aguardavam consulta médica ou de enfermagem; (3) mulheres que aguardavam atendimento para qualquer outra atividade na UBS. Não foi possível selecionar aleatoriamente as mulheres, tendo em vista que há diversas combinações de organização de consultas para coleta de Papanicolaou nas UBS, como agendamento de todas no mesmo horário ou demanda aberta, sem qualquer tipo de agendamento.

A coleta de dados foi realizada por meio de entrevista face a face por pesquisadoras graduadas na área da saúde. As entrevistas foram realizadas por meio de um instrumento estruturado com perguntas sobre o perfil sociodemográfico e reprodutivo e mediante o calendário contraceptivo, utilizado nas pesquisas DHS 2 . Trata-se de um histórico retrospectivo mês a mês sobre gestações, nascimentos, abortamentos e episódios de uso de métodos contraceptivos referentes aos cinco anos anteriores à entrevista.

Para cada episódio de uso de método contraceptivo descontinuado, as mulheres foram questionadas sobre a razão que as levou a descontinuar o uso. Essas razões foram descritas por meio de proporção e foram categorizadas em dois tipos: (a) "não relacionadas ao método contraceptivo", no caso de descontinuidade porque queria engravidar, tinha dificuldade de engravidar ou já estava na menopausa, tinha relações sexuais pouco frequentes e descontinuou por separação do parceiro; e (b) "relacionadas ao método contraceptivo", quando as razões foram porque engravidou enquanto usava o método, porque queria um método mais eficaz, por conta dos efeitos colaterais, porque o método era inconveniente para usar, porque tinha preocupação com sua saúde, por oposição do marido/parceiro, por problemas de acesso e custo do método e outras/não soube responder 2. Adicionalmente, foi usado um instrumento para averiguar o perfil sociodemográfico e reprodutivo das mulheres, cujas variáveis aqui descritas são a idade (18-24, 25-34 e 35 anos e mais), a escolaridade (até 8 anos de estudo, 9-11 anos e 12 anos e mais), a cor da pele (branca, parda, preta e amarela/indígena), o grupo socioeconômico (A e B, C, D e E, classificação realizada de acordo com o Critério Brasil 2015 da Associação Brasileira de Empresas de Pesquisa 16), a religião (católica, evangélica, nenhuma e outras), o exercício de trabalho remunerado (não e sim), a união conjugal (não e sim), o desejo de ter (mais) filhos (não e sim), gravidez atual (não e sim) e uso de método contraceptivo na última relação sexual (nenhum, contraceptivo hormonal oral, injetável, preservativo masculino, laqueadura, vasectomia, dispositivo intrauterino - DIU, comportamental e outros).

Probabilidades de sobrevivência estimadas pelo método Kaplan-Meier foram utilizadas para estimar as taxas acumuladas de descontinuidade no uso dos métodos contraceptivos, definidas como o intervalo de tempo entre o início do uso do método e o momento em que foi descontinuado. O segmento de uso do método contraceptivo é a unidade de análise e foi definido como o período de tempo contado a partir do mês em que o uso do método é iniciado até o mês em que é descontinuado, por abandono, troca ou falha. Uma mulher pode ter contribuído com vários segmentos de uso de métodos, caso tenha interrompido e iniciado o seu uso várias vezes durante o período coberto pelo calendário contraceptivo (ou seja, as mulheres puderam contribuir com novos episódios de uso do mesmo método contraceptivo) ou com nenhum segmento, caso não tenha usado qualquer método. Este estudo adotou estratégias metodológicas em consonância com estudos internacionais que avaliam descontinuidades contraceptivas por meio do calendário contraceptivo 2,17. Dessa forma, segmentos de não uso de métodos, assim como os segmentos de uso de métodos irreversíveis, de gravidez, de parto e de abortamento foram tratados como censura. Somente segmentos de uso de métodos iniciados durante o calendário (novos segmentos) foram analisados, tendo em vista que a duração do uso dos métodos iniciados antes do início do calendário é desconhecida (censura à esquerda). Segmentos de uso de métodos que ainda estavam ocorrendo no momento da entrevista foram tratados como censura à direita. Os três meses anteriores à entrevista foram censurados porque uma mulher poderia estar grávida sem ainda o saber.

São apresentadas as taxas acumuladas de descontinuidade total (abandono, troca para um método menos e mais eficaz) segundo os segmentos de uso de métodos contraceptivos, por tipo de método. 
Posteriormente, são apresentadas as razões para descontinuar o uso do método tanto por motivos não relacionadas quanto por razões relacionadas ao método. Também são apresentadas as taxas acumuladas de descontinuidade por abandono, separadamente por grupo de mulheres que interromperam o uso do método por qualquer razão e por grupo de mulheres que abandonaram o uso por razões relacionadas ao método, isto é, considerando apenas aquelas que tinham necessidade de contracepção. Foi considerado abandono quando, em um segmento de uso, a mulher parou de usar o método e não usou nenhum no mês posterior. São apresentadas também as taxas acumuladas de descontinuidade por troca, identificada quando a mulher parou de usar o método contraceptivo e usou outro no mês posterior. A análise foi estratificada por tipo, sendo troca para método menos eficaz e troca para método mais eficaz 18, independentemente da razão. Todas as taxas foram calculadas aos 12 meses de uso do contraceptivo hormonal oral, do preservativo masculino e de contraceptivo hormonal injetável. Foi também descrita a proporção de mulheres segundo suas razões para descontinuar o uso do método, por capital e por tipo de método. As taxas de descontinuidade por falhas não foram estimadas. Todas as análises foram conduzidas no Stata 14.0 (https://www.stata.com).

O projeto de pesquisa foi aprovado por Comitê de Ética em Pesquisa, tendo sido respeitados todos os preceitos, de acordo com a Resolução no 466/2012, do Conselho Nacional de Saúde (CAAE 40558714.8.3001.0086 e 40558714.8.0000.5392). As entrevistas foram realizadas em local privativo, como consultórios ou salas de reunião, no momento em que as mulheres preferissem, ou seja, antes ou após o atendimento na UBS. Elas também puderam interromper a participação no estudo a qualquer momento e deixar de responder às perguntas que quisessem, sem qualquer prejuízo ao seu atendimento. O Termo de Consentimento Livre e Esclarecido foi assinado por todas as participantes.

\section{Resultados}

Ao todo, 1.030 mulheres foram entrevistadas em São Paulo, 508 em Aracaju e 513 em Cuiabá ( $\mathrm{N}=$ 2.051). Elas tinham 30,1 anos de idade, em média ( $D P=7,9)$, e quase um terço tinha entre 18 e 24 anos de idade. Apenas 14,7\% tinham escolaridade igual ou maior que 12 anos. A maioria classificouse como parda (52,3\%), pertencia ao grupo socioeconômico C $(63,8 \%)$, relatou alguma religião, como católica (46,4\%) ou evangélica (36,6\%), e estava em união conjugal (73,6\%). Em relação à história reprodutiva, $87 \%$ tiveram, pelo menos, um filho. Mais da metade $(58,4 \%)$ respondeu que não queria ter (mais) filhos. Quase um quarto estava grávida no momento da entrevista (24,5\%). Dentre as que relataram não estar grávidas, os métodos mais usados na última relação sexual foram o contraceptivo hormonal oral (30,2\%), o injetável $(18,8 \%)$ e o preservativo masculino $(17,8 \%)$ (Tabela 1$)$.

Foram analisados 3.209 segmentos de uso de métodos contraceptivos, sendo 1.757 referentes às mulheres de São Paulo, 751 de Aracaju e 701 de Cuiabá, que totalizaram 74.236 meses de observação. Quase um terço de todos os segmentos de uso de métodos (30\% ou $\mathrm{n}=962)$ finalizou em abandono do método em 12 meses de observação; 10,7\% finalizaram em troca para método contraceptivo mais eficaz ( $n=345) ; 9,8 \%$ finalizaram em troca para método contraceptivo menos eficaz $(n=316)$; e 15,6\% em falha, ou seja, em uma gravidez enquanto se usava o método $(\mathrm{n}=500)$. Pouco mais de um terço dos segmentos de uso de métodos contraceptivos não foi descontinuado no período $(33,9 \%$ ou $\mathrm{n}=1.086)$ (dados não apresentados em tabela).

A Tabela 2 mostra que a taxa de descontinuidade no uso do contraceptivo hormonal oral em 12 meses de uso foi de $24,5 \%$, mais baixa que a taxa do preservativo masculino (39\%) e do contraceptivo hormonal injetável (33,5\%). As taxas de descontinuidade do preservativo masculino foram $45,8 \%$, 30,2\% e 31,4\% em São Paulo, Aracaju e Cuiabá, respectivamente, ao passo que as do contraceptivo hormonal injetável foram 39,7\%, 33,6\% e 21,5\%, nessa mesma ordem.

A principal razão para se descontinuar o uso do método contraceptivo foi por querer engravidar (20,8\%). Um total de $20 \%$ das mulheres engravidou enquanto usava algum método, e essa proporção chegou a 28,7\% em Cuiabá e 25,7\% entre usuárias do preservativo masculino. Razões para descontinuar o uso do método contraceptivo não relacionadas aos próprios métodos totalizaram 30,7\%, e as razões relacionadas ao método totalizaram 59,1\% (outras razões ou "não sabe" corresponderam a 10,2\%). A principal razão para descontinuar o uso do contraceptivo hormonal oral foi por querer engravidar $(25,4 \%)$. No caso do preservativo masculino, a principal razão foi por querer um método 


\section{Tabela 1}

Características sociodemográficas e da história reprodutiva das mulheres usuárias de unidades básicas de saúde. São Paulo (São Paulo), Aracaju (Sergipe) e Cuiabá (Mato Grosso), Brasil, 2015-2017.

\begin{tabular}{|c|c|c|}
\hline Variável & $\mathbf{n}$ & $\%$ \\
\hline \multicolumn{3}{|l|}{ Município } \\
\hline São Paulo & 1.030 & 50,2 \\
\hline Aracaju & 508 & 24,8 \\
\hline Cuiabá & 513 & 25,0 \\
\hline \multicolumn{3}{|l|}{ Idade (anos) } \\
\hline $18-24$ & 607 & 29,6 \\
\hline $25-34$ & 839 & 40,9 \\
\hline 35 e mais & 605 & 29,5 \\
\hline \multicolumn{3}{|l|}{ Escolaridade (anos) } \\
\hline Até 8 & 555 & 27,1 \\
\hline $9-11$ & 1.194 & 58,2 \\
\hline 12 e mais & 302 & 14,7 \\
\hline \multicolumn{3}{|l|}{ Cor da pele * } \\
\hline Branca & 506 & 24,7 \\
\hline Parda & 1.072 & 52,3 \\
\hline Preta & 360 & 17,6 \\
\hline Amarela/Indígena & 110 & 5,4 \\
\hline \multicolumn{3}{|l|}{ Grupo socioeconômico } \\
\hline$A$ e $B$ & 295 & 14,4 \\
\hline C & 1.039 & 63,8 \\
\hline$D$ e $E$ & 447 & 21,8 \\
\hline \multicolumn{3}{|l|}{ Religião } \\
\hline Católica & 951 & 46,4 \\
\hline Evangélica & 751 & 36,6 \\
\hline Nenhuma & 256 & 12,5 \\
\hline Outras ** & 93 & 4,5 \\
\hline \multicolumn{3}{|l|}{ Exerce trabalho remunerado } \\
\hline Não & 984 & 48,0 \\
\hline Sim & 1.067 & 52,0 \\
\hline \multicolumn{3}{|l|}{ União conjugal } \\
\hline Não & 542 & 26,4 \\
\hline Sim & 1.509 & 73,6 \\
\hline \multicolumn{3}{|l|}{ Número de filhos } \\
\hline Nenhum & 235 & 13,0 \\
\hline 1 & 747 & 41,4 \\
\hline 2 & 485 & 26,9 \\
\hline 3 e mais & 336 & 18,7 \\
\hline \multicolumn{3}{|l|}{ Intenção de ter (mais) filhos } \\
\hline Quer ter (mais) filhos & 719 & 35,0 \\
\hline Não quer ter (mais) filhos & 1.197 & 58,4 \\
\hline Não sabe & 135 & 6,6 \\
\hline \multicolumn{3}{|l|}{ Gravidez atual } \\
\hline Não & 1.508 & 73,5 \\
\hline Sim & 502 & 24,5 \\
\hline Não sabe & 41 & 2,0 \\
\hline
\end{tabular}

(continua) 
Tabela 1 (continuação)

\begin{tabular}{lcc}
\hline Variável & $\mathbf{n}$ & $\%$ \\
\hline Uso de método contraceptivo *** & 322 & 21,4 \\
Nenhum & 456 & 30,2 \\
Contraceptivo hormonal oral & 283 & 18,8 \\
Contraceptivo hormonal injetável & 268 & 17,8 \\
Preservativo masculino & 74 & 4,9 \\
Laqueadura & 26 & 1,7 \\
Vasectomia & 32 & 2,1 \\
DIU & 41 & 2,7 \\
Comportamental & 6 & 0,4 \\
Outros \# & 2.051 & 100,0 \\
Total & & \\
\hline
\end{tabular}

* Não há informação de todas as mulheres;

** Espírita, judia, umbandista, candomblecista;

*** Consideradas somente mulheres que não estavam grávidas $(n=1.508)$;

\# LAM (método da lactação e amenorréia), adesivo, camisinha feminina, implante.

\section{Tabela 2}

Probabilidades acumuladas de descontinuidade total de métodos anticoncepcionais nos 12 meses de uso e respectivos intervalos de $95 \%$ de confiança (IC95\%), por tipo de método contraceptivo. São Paulo (São Paulo), Aracaju (Sergipe) e Cuiabá (Mato Grosso), Brasil, 2015-2017.

\begin{tabular}{|c|c|c|c|c|c|c|c|c|}
\hline \multirow[t]{3}{*}{ Métodos contraceptivos } & \multicolumn{8}{|c|}{ Taxa de descontinuidade acumulada por 100 episódios } \\
\hline & \multicolumn{2}{|c|}{ Total $(\mathrm{N}=3.209)$} & \multicolumn{2}{|c|}{ São Paulo $(n=1.757)$} & \multicolumn{2}{|c|}{ Aracaju $(n=751)$} & \multicolumn{2}{|c|}{ Cuiabá $(n=701)$} \\
\hline & $\%$ & IC95\% & $\%$ & IC95\% & $\%$ & IC95\% & $\%$ & IC95\% \\
\hline Contraceptivo hormonal oral & 24,5 & $21,4-28,1$ & 29,7 & $24,9-35,5$ & 19,7 & $14,3-27,2$ & 18,5 & $13,4-25,4$ \\
\hline Preservativo masculino & 39,0 & $33,6-45,3$ & 45,8 & $38,0-55,2$ & 30,2 & $21,6-42,2$ & 31,4 & $21,7-45,5$ \\
\hline Contraceptivo hormonal injetável & 33,5 & $28,2-39,8$ & 39,7 & $31,6-49,7$ & 33,6 & $23,9-47,3$ & 21,5 & $14,2-32,7$ \\
\hline
\end{tabular}

mais eficaz (30,6\%) e, no caso do contraceptivo hormonal injetável, por conta dos efeitos colaterais (27\%) (Tabela 3).

Independentemente das razões relatadas pelas mulheres para descontinuarem o uso do método adotado, 13,6\% das usuárias de contraceptivo hormonal injetável o abandonaram até 12 meses de uso, sendo o mesmo observado entre 9,6\% das usuárias do contraceptivo oral e 10,6\% entre usuárias do preservativo masculino. Quando consideradas apenas as razões relacionadas aos métodos, a taxa de abandono foi de 11,4\% entre usuárias do contraceptivo hormonal injetável, de 7\% entre usuárias do contraceptivo oral e de 5,4\% entre usuárias do preservativo masculino, padrão similar ao que foi verificado em todas as capitais (Tabela 4).

O método contraceptivo mais descontinuado por troca para outro mais eficaz foi o preservativo masculino, alcançando $15,9 \%$ aos 12 meses de uso do método. As taxas de troca para método mais eficaz entre usuárias do preservativo masculino foram de 20\% em São Paulo, de 9,8\% em Aracaju e de 12,4\% em Cuiabá. Por sua vez, a troca para método menos eficaz foi maior entre as usuárias de contraceptivo hormonal injetável, alcançando 16,3\%, também aos 12 meses de uso do método, sendo 21,8\% em São Paulo, 14,5\% em Aracaju e 7,8\% em Cuiabá (Tabela 5). 


\section{Tabela 3}

Distribuição percentual das razões para descontinuidade contraceptiva entre mulheres usuárias de unidades básicas de saúde. São Paulo (São Paulo) Aracaju (Sergipe) e Cuiabá (Mato Grosso), Brasil, 2015-2017.

\begin{tabular}{|c|c|c|c|c|c|c|c|}
\hline \multirow{2}{*}{$\begin{array}{l}\text { Razões para descontinuar o uso do método } \\
\text { contraceptivo }\end{array}$} & \multirow[t]{2}{*}{ Total } & \multicolumn{3}{|c|}{ Capital } & \multicolumn{3}{|c|}{ Método em uso } \\
\hline & & São Paulo & Aracaju & Cuiabá & $\begin{array}{l}\text { Contraceptivo } \\
\text { hormonal oral }\end{array}$ & $\begin{array}{l}\text { Preservativo } \\
\text { masculino }\end{array}$ & $\begin{array}{c}\text { Contraceptivo } \\
\text { hormonal } \\
\text { injetável }\end{array}$ \\
\hline \multicolumn{8}{|l|}{ Não relacionadas aos métodos contraceptivos } \\
\hline Quis engravidar & 20,8 & 20,8 & 17,5 & 24,7 & 25,4 & 13,5 & 24,4 \\
\hline Sexo infrequente & 6,7 & 7,0 & 8,1 & 4,2 & 6,8 & 7,7 & 6,4 \\
\hline Separação & 2,3 & 2,2 & 4,3 & 0,5 & 2,1 & 1,6 & 3,1 \\
\hline Dificuldade de engravidar/Menopausa & 0,9 & 0,9 & 1,1 & 0,5 & 1,2 & 0,7 & 0,4 \\
\hline \multicolumn{8}{|l|}{ Relacionadas aos métodos contraceptivos } \\
\hline Engravidou enquanto usava & 20,0 & 17,4 & 18,8 & 28,7 & 18,6 & 25,7 & 8,4 \\
\hline Efeitos colaterais & 14,9 & 16,2 & 15,8 & 10,3 & 19,4 & 0,9 & 27,0 \\
\hline Preocupação com a saúde & 4,6 & 4,9 & 4,7 & 3,5 & 5,9 & 1,2 & 6,6 \\
\hline Queria método mais eficaz & 12,8 & 13,2 & 11,7 & 12,9 & 5,2 & 30,6 & 3,1 \\
\hline Inconveniente para usar & 3,3 & 3,2 & 3,4 & 3,8 & 2,2 & 6,5 & 2,4 \\
\hline Falta de acesso & 1,9 & 2,8 & 1,1 & 0,5 & 1,2 & 0,2 & 6,2 \\
\hline Custo & 0,5 & 0,6 & 0,8 & - & 0,6 & 0,2 & 0,7 \\
\hline Marido se opôs & 1,1 & 0,8 & 1,7 & 0,9 & 0,1 & 3,0 & 0,7 \\
\hline Não sabe/Outros & 10,2 & 10,0 & 11,0 & 9,5 & 11,3 & 8,2 & 10,6 \\
\hline Número de episódios descontinuados & 2.123 & 1.177 & 470 & 476 & 912 & 589 & 461 \\
\hline
\end{tabular}

\section{Tabela 4}

Probabilidades acumuladas de abandono de métodos anticoncepcionais nos 12 meses de uso e respectivos intervalos de 95\% de confiança (IC95\%), por tipo de método contraceptivo, de acordo com as razões para o abandono. São Paulo (São Paulo), Aracaju (Sergipe) e Cuiabá (Mato Grosso), Brasil, 2015-2017.

\begin{tabular}{|c|c|c|c|c|c|c|c|c|}
\hline \multirow[t]{3}{*}{ Métodos contraceptivos } & \multicolumn{8}{|c|}{ Taxa de descontinuidade acumulada por 100 episódios } \\
\hline & \multicolumn{2}{|c|}{ Total } & \multicolumn{2}{|c|}{ São Paulo } & \multicolumn{2}{|c|}{ Aracaju } & \multicolumn{2}{|c|}{ Cuiabá } \\
\hline & $\%$ & IC95\% & $\%$ & IC95\% & $\%$ & IC95\% & $\%$ & IC95\% \\
\hline \multicolumn{9}{|c|}{ Descontinuidade por abandono (todas as razões) } \\
\hline Contraceptivo hormonal oral & 9,6 & $7,7-12,0$ & 10,8 & $8,1-14,5$ & 9,1 & $5,6-14,6$ & 7,8 & $4,8-12,7$ \\
\hline Preservativo masculino & 10,6 & $8,0-14,1$ & 12,7 & $8,9-18,0$ & 11,6 & $6,7-19,9$ & 3,4 & $1,1-10,5$ \\
\hline Contraceptivo hormonal injetável & 13,6 & $10,3-17,7$ & 15,2 & $10,6-21,9$ & 15,2 & $9,2-25,3$ & 8,8 & $4,6-17,0$ \\
\hline \multicolumn{9}{|c|}{ Descontinuidade por abandono (razões relacionadas ao método) } \\
\hline Contraceptivo hormonal oral & 7,0 & $5,2-9,4$ & 8,3 & $5,6-12,4$ & 7,3 & $4,0-13,1$ & 4,3 & $2,0-8,9$ \\
\hline Preservativo masculino & 5,4 & $3,5-8,4$ & 6,7 & $3,9-11,5$ & 6,4 & $2,8-14,1$ & 1,2 & $0,2-8,7$ \\
\hline Contraceptivo hormonal injetável & 11,4 & $8,1-16,0$ & 14,2 & $9,2-21,7$ & 9,8 & $4,7-20,6$ & 6,9 & $2,9-16,6$ \\
\hline
\end{tabular}




\section{Tabela 5}

Probabilidades acumuladas de troca para métodos anticoncepcionais mais e menos eficazes nos 12 meses de uso e respectivos intervalos de $95 \%$ de confiança, por tipo de método contraceptivo, entre mulheres em geral e aquelas com necessidade de contracepção. São Paulo (São Paulo), Aracaju (Sergipe) e Cuiabá (Mato Grosso), Brasil, 2015-2017.

\begin{tabular}{|c|c|c|c|c|c|c|c|c|}
\hline \multirow[t]{3}{*}{ Métodos contraceptivos } & \multicolumn{8}{|c|}{ Taxa de descontinuidade acumulada por 100 episódios } \\
\hline & \multicolumn{2}{|c|}{ Total } & \multicolumn{2}{|c|}{ São Paulo } & \multicolumn{2}{|c|}{ Aracaju } & \multicolumn{2}{|c|}{ Cuiabá } \\
\hline & $\%$ & IC95\% & $\%$ & IC95\% & $\%$ & IC95\% & $\%$ & IC95\% \\
\hline \multicolumn{9}{|c|}{ Descontinuidade por troca para método mais eficaz } \\
\hline Contraceptivo hormonal oral & 3,2 & $2,2-4,7$ & 4,1 & $2,6-6,6$ & 2,1 & $0,8-5,7$ & 2,4 & $1,0-5,8$ \\
\hline Preservativo masculino & 15,9 & $12,6-20,1$ & 20,0 & $15,1-26,6$ & 9,8 & $5,4-17,7$ & 12,4 & $6,9-22,4$ \\
\hline Contraceptivo hormonal injetável & 0,5 & $0,1-2,1$ & 0,5 & $0,1-3,9$ & 1,0 & $0,1-6,8$ & - & - \\
\hline \multicolumn{9}{|c|}{ Descontinuidade por troca para método menos eficaz } \\
\hline Contraceptivo hormonal oral & 5,1 & $3,8-6,9$ & 6,7 & $4,5-9,6$ & 4,8 & $2,5-9,2$ & 2,4 & $1,0-5,8$ \\
\hline Preservativo masculino & 1,4 & $0,6-3,0$ & 1,7 & $0,3-4,5$ & 1,7 & $0,4-6,9$ & - & - \\
\hline Contraceptivo hormonal injetável & 16,3 & $12,8-20,9$ & 21,8 & $16,0-29,6$ & 14,5 & $8,6-24,5$ & 7,8 & $3,9-15,6$ \\
\hline
\end{tabular}

\section{Discussão}

Este estudo utilizou o calendário contraceptivo para guiar entrevistas com mulheres usuárias de UBS de três capitais brasileiras, de diferentes regiões do país, com o intuito de estimar as taxas de descontinuidade no uso do contraceptivo hormonal oral, do preservativo masculino e de contraceptivo hormonal injetável nos 12 meses posteriores ao início de uso do método. Nossos resultados mostraram algo que já era esperado: altas taxas de descontinuidade no uso do contraceptivo hormonal oral, do preservativo masculino e de contraceptivo hormonal injetável entre as mulheres residentes nessas capitais, tendo em vista que pouco mais de um terço das usuárias do preservativo masculino e de contraceptivo hormonal injetável, e um quarto, no caso do contraceptivo oral, descontinuaram o uso do método em 12 meses de uso, independentemente das razões que motivaram a descontinuidade.

A comparação dos nossos achados com outros estudos brasileiros é delicada, pelo fato de que analisamos apenas três tipos de métodos contraceptivos. De toda forma, parece que as taxas de descontinuidade dos métodos contraceptivos reversíveis permaneceram altas nas duas últimas décadas, quando comparadas às estimativas conduzidas com os dados da PNDS/1996 8,14. A comparação com estudos mais recentes é ainda mais limitada, pois o calendário contraceptivo não foi usado 13,15.

Os métodos contraceptivos analisados neste estudo requerem uma atenção maior da mulher/casal para o uso e não exigem a ação de um profissional de saúde para sua interrupção. Portanto, tendem a ser os mais descontinuados 10 . Apesar de ser considerado o principal aspecto que determina a ocorrência de descontinuidades, o tipo de método contraceptivo explica o fenômeno apenas em parte. É preciso considerar também o papel da insatisfação ou as dificuldades de manejo com o método ${ }^{19}$, das relações que se estabelecem com parcerias afetivo-sexuais 3 e da adequabilidade da atenção ao planejamento reprodutivo, incluindo o aconselhamento contraceptivo ${ }^{10}$. Parece, portanto, que a atenção em contracepção ainda precisa avançar em termos de qualidade da assistência para subsidiar a continuidade no uso dos métodos contraceptivos.

Cabe destacar que analisamos separadamente três tipos de descontinuidades: abandono, troca por método contraceptivo menos eficaz e troca por método mais eficaz. Entender suas diferenças é importante, porque cada um impacta de forma peculiar na ocorrência da gravidez não intencional e, consequentemente, na trajetória reprodutiva. $\mathrm{O}$ abandono e a troca para método menos eficaz são eventos que, de certa forma, requerem mais atenção, por ocasionarem situações em que as mulheres ficam vulneráveis à gravidez não intencional, ao passo que a troca para método contraceptivo mais eficaz pode ser considerada um evento positivo 6,8,20. Todavia, o período de adaptação e de familiaridade ao método contraceptivo, primordial para sua continuidade, não deve ser desconsiderado. 
Soma-se a isso o fato de que a descontinuidade é comum quando se usa o método pela primeira vez, ou seja, mesmo adotando um método mais eficaz, a mulher pode vir a descontinuá-lo por falta de adaptação ou por inexperiência de uso 8,14,15,21. A troca do método contraceptivo é mais frequente nos países onde os métodos hormonais, como contraceptivo hormonal oral e injetável, são comumente usados, como é o caso do Brasil e de demais países da América Latina, do que nos países onde predomina 6 o uso de métodos reversíveis de longa duração (LARC), ou seja, dispositivo intrauterino, sistema intrauterino e implantes subdérmicos.

Especificamente no que concerne ao contraceptivo hormonal oral, é preciso ressaltar que esse é o método mais utilizado pelas mulheres brasileiras 1 . Apesar da alta prevalência e de ampla disponibilidade 17, sua descontinuidade é frequente, principalmente por conta dos efeitos colaterais 2,8,9,12,13,15,21, o que condiz com nossos achados, tendo em vista que um quinto das mulheres descontinuou o contraceptivo oral por essa razão. A descontinuidade no uso do contraceptivo hormonal oral, quando ainda há necessidade de contracepção, parece indicar que as usuárias não estavam preparadas para lidar com os seus efeitos colaterais ou sequer sabiam que eles são frequentes, o que sugere inadequação no aconselhamento em contracepção.

Foi observada troca de contraceptivo oral e de injetável para método menos eficaz. A restrição no acesso a métodos mais eficazes pode explicar, em parte, este resultado ${ }^{6}$. É provável que a mulher possa até ter desejado um método mais eficaz, como LARC ou laqueadura, mas não teve a oportunidade para troca e ficou sem escolha, uma vez que o acesso a eles é permeado por barreiras institucionais 22,23. Isso significa que há a necessidade de se ampliar a oferta dos LARC no cenário reprodutivo brasileiro, pois são métodos reversíveis com menor probabilidade de serem descontinuados, justamente por não dependerem de ação regular da mulher para sua utilização 24 e por serem os que geram maior satisfação, segundo relato das usuárias 25 . Além disso, os LARC são métodos seguros para a maior parte das mulheres, incluindo adolescentes e nulíparas, além de serem altamente eficazes, com taxa de falha similar à de métodos permanentes, como esterilização feminina e masculina, com a vantagem de serem reversíveis, o que permite a interrupção de seu uso quando a mulher desejar 24 .

Os contraceptivos hormonais injetáveis também apresentaram alta taxa de descontinuidade. Inclusive, em vários estudos, esse foi o método mais descontinuado 14,21,26,27. A alta taxa de descontinuidade desse método pode estar associada à dificuldade de obtê-lo, o que envolve, por exemplo, a obrigatoriedade de comparecer à unidade de saúde mensal ou trimestralmente e a necessidade de intervenção de um profissional de saúde para sua aplicação 28 , mas esse não parece ter sido o caso entre as mulheres deste estudo, dado que foram os efeitos colaterais a principal razão para descontinuá-lo (embora problemas no acesso também tenham sido relatados como razão para interrompê-lo). Por sua vez, o abandono dos métodos hormonais, quando ainda há necessidade de contracepção, pode representar apenas um período de pausa, até que a mulher consiga outro método que seja mais adequado a sua necessidade 27 , que pode ser outro tipo de contraceptivo oral ou injetável. Há, ainda, a possibilidade de que o novo método seja para "descansar o corpo" dos efeitos hormonais, como foi relatado por algumas mulheres do presente estudo (dados não apresentados).

Estudos também observaram que o preservativo masculino foi frequentemente descontinuado, não obstante a taxa de descontinuidade ter variado a depender do contexto 2,9,29. Por exemplo, as taxas de descontinuidade no uso do preservativo masculino observadas neste estudo foram menores do que as observadas em outros países, como Estados Unidos, França, entre outros 2,9,27. É importante notar que, apesar das altas taxas de descontinuidade total, a troca para método mais eficaz foi expressiva entre as usuárias do preservativo masculino. Pode-se considerar que esse é um resultado positivo, pois, ao optar por método mais eficaz, a mulher/casal aumenta a possibilidade de se prevenir de uma gravidez, visto que o preservativo masculino é o método com maior probabilidade de falha, se comparado aos demais métodos contraceptivos modernos 18. Uma das nossas limitações é que não investigamos para qual método migraram nem se havia qualquer preocupação com a prevenção de infecções sexualmente transmissíveis nessas transições.

Pode ser que o preservativo masculino esteja sendo usado mais como "reforço" de outros métodos contraceptivos, sendo de caráter provisório. A mulher ou o casal, quando têm oportunidade ou necessidade, trocam o método, geralmente por um mais eficaz 30,31. Estudo que analisou a dinâmica da troca entre preservativo masculino e contraceptivos hormonais observou que as mulheres experienciam alternâncias importantes entre os dois métodos 30 . Isso denota que é relevante envolver o parceiro 
na adoção e na continuação do uso do preservativo masculino, visto que as usuárias reportaram a inconveniência para usá-lo e a oposição do parceiro como razões para descontinuar a sua utilização, principalmente por conta da ideia de que há interferência no prazer e na satisfação sexual 21.

Este estudo apresenta algumas limitações. Uma delas é que não foi averiguada a consistência do uso dos métodos nem se o uso era correto, tendo em vista que este não foi nosso objetivo. Tampouco foram analisadas as taxas de descontinuidade de outros métodos reversíveis, justamente por conta do baixo número de segmentos de uso. Outra limitação importante é o possível viés de memória, que pode se manifestar de forma diferente a depender do contraceptivo em uso e das razões de descontinuidade, justamente por conta do longo período (cinco anos) a que o estudo se refere. Por outro lado, as potencialidades incluem métodos de pesquisa comparáveis a estudos do tipo DHS conduzidos em inúmeros países, como o uso do calendário contraceptivo, reconhecidamente válido 4,10,17,27. Ainda, nossos dados contemplam informações sobre mulheres residentes em diferentes regiões do país e nossos achados mostram que o contraceptivo oral, o preservativo masculino e o injetável podem ter padrões distintos de descontinuidades nas capitais analisadas, o que aponta a necessidade de futuros estudos que possam explicar tais diferenças e subsidiar diferentes estratégias de atenção em contracepção.

Por fim, ressalta-se que é imperativo melhorar a qualidade da atenção ao planejamento reprodutivo no Sistema Único de Saúde (SUS), por meio da oferta de adequado aconselhamento contraceptivo, contemplando os critérios de elegibilidade e intenção reprodutiva de mulheres e de seus parceiros 24 , seguimento das usuárias quando iniciam o uso de um método, no intuito de propiciar condições para alcançar as preferências reprodutivas 4 e oferta ampliada dos LARC, principalmente o DIU de cobre, que está disponível nas UBS. O DIU de cobre não tem os efeitos colaterais dos métodos hormonais, pode ser usado por mulheres em situação de pós-parto e de pós-abortamento e não necessita de um profissional médico para sua inserção, tendo em vista que, no Brasil 32, enfermeiros treinados e capacitados têm competência legal para inseri-lo e retirá-lo.

\section{Colaboradores}

A. L. V. Borges concebeu, planejou e implementou o estudo, além de ter analisado os dados e redigido o manuscrito. C. B. N. Chofakian concebeu o estudo, analisou os dados e redigiu o manuscrito. O. A. Viana e E. A. Divino implementaram o estudo, administraram a coleta de dados, analisaram os dados e redigiram o manuscrito.

\section{Informações adicionais}

ORCID: Ana Luiza Vilela Borges (0000-0002-28071762); Christiane Borges do Nascimento Chofakian (0000-0002-5953-3296); Osmara Alves Viana (0000-0001-6360-861X); Eveline do Amor Divino (0000-0003-0037-0557).

\section{Agradecimentos}

Fundação de Amparo à Pesquisa do Estado de São Paulo (2014/02447-5) e Conselho Nacional de Desenvolvimento Científico e Tecnológico (440577/2014-4). 


\section{Referências}

1. Departamento de Ciência e Tecnologia, Secretaria da Ciência, Tecnologia e Insumos Estratégicos, Ministério da Saúde. PNDS 2006. Pesquisa Nacional de Demografia e Saúde da Criança e da Mulher: relatório. http://bvms. saude.gov.br/bvs/publicacoes/relatório_ pnds_2006.pdf (acessado em 29/Set/2019).

2. Bradley SEK, Schwandt HM, Khan S. Levels, trends, and reasons for contraceptive discontinuation. DHS Analytical Studies, 20. https:// www.dhsprogram.com/publications/publica tion-as20-analytical-studies.cfm (acessado em 29/Set/2019).

3. Castle S, Askew I. Contraceptive discontinuation: reasons, challenges, and solutions. http:// ec2-54-210-230-186.compute-1.amazonaws. com/wp-content/uploads/2015/12/FP2020_ ContraceptiveDiscontinuation_SinglePage_ Final_12.08.15.pdf (acessado em 29/Set/2019).

4. Jain AK, Winfrey W. Contribution of contraceptive discontinuation to unintended births in 36 developing countries. Stud Fam Plann 2017; 48:269-78.

5. Theme-Filha MM, Baldisserotto ML, Fraga ACS, Ayers S, Gama SG, Leal MC. Factors associated with unintended pregnancy in Brazil: cross-sectional results from the Birth in Brazil National Survey, 2011/2012. Reprod Health 2016; 13 Suppl 3:118.

6. Ali MM, Cleland J. Contraceptive switching after method-related discontinuation: levels and differentials. Stud Fam Plann 2010; 41:129-33.

7. Modey EJ, Aryeetey R, Adanu R. Contraceptive discontinuation and switching among Ghanaian women: evidence from the Ghana Demographic and Health Survey, 2008. Afr J Reprod Health 2014; 18:84-92.

8. Ali MM, Cleland J. Oral contraceptive discontinuation and its aftermath in 19 developing countries. Contraception 2010; 81:22-9.

9. Moreau C, Bouyer J, Bajos N, Rodríguez G, Trussell J. Frequency of discontinuation of contraceptive use: results from a French population-based cohort. Hum Reprod 2009; 24:1387-92.

10. Ali MM, Cleland J, Shah IH. Causes and consequences of contraceptive discontinuation: evidence from 60 Demographic and Health Surveys. http://apps.who.int/iris/bitstre am/10665/75429/1/9789241504058_eng.pdf (acessado em 29/Set/2019).

11. Raine TR, Foster-Rosales A, Upadhyay UD, Boyer CB, Brown BA, Sokoloff A, et al. Oneyear contraceptive continuation and pregnancy in adolescent girls and women initiating hormonal contraceptives. Obstet Gynecol 2011; $117(2$ Pt 1):363-71.

12. Peipert JF, Zhao Q, Allsworth JE, Petrosky E, Madden T, Eisenberg D, et al. Continuation and satisfaction of reversible contraception. Obstet Gynecol 2011; 117:1105-13.
13. Ferreira JM, Nunes FR, Modesto WS, Gonçalves MP, Bahamondes L. Reasons for Brazilian women to switch from different contraceptives to long-acting reversible contraceptives. Contraception 2014; 89:17-21.

14. Leite IC, Gupta N. Assessing regional differences in contraceptive discontinuation, failure and switching in Brazil. Reprod Health 2007; 4:1-10.

15. Bahamondes L, Pinho F, Melo NR, Oliveira E, Bahamondes MV. Fatores associados à descontinuação do uso de anticoncepcionais orais combinados. Rev Bras Ginecol Obstet 2011; 33:303-9.

16. Associação Brasileira de Empresas de Pesquisa. Critério Brasil 2015. http://www.abep.org/ criterio-brasil (acessado em 07/Jun/2020).

17. Barden-O'Fallon J, Speizer IS, Calhoun LM, Corroon M. Women's contraceptive discontinuation and switching behavior in urban Senegal, 2010-2015. BMC Womens Health 2018; 18:35.

18. Trussell J. Contraceptive failure in the United States. Contraception 2011; 83:397-404.

19. Littlejohn KE. Hormonal contraceptive use and discontinuation because of dissatisfaction: differences by race and education. Demography 2012; 49:1433-52.

20. Curtis S, Evens E, Sambisa W. Contraceptive discontinuation and unintended pregnancy: an imperfect relationship. Int Perspect Sex Reprod Health 2011; 37:58-66.

21. Moreau C, Cleland K, Trussell J. Contraceptive discontinuation attributed to method dissatisfaction in the United States. Contraception 2007; 76:267-72.

22. Caetano AJ. Esterilização cirúrgica feminina no Brasil, 2000 a 2006: aderência à lei de planejamento familiar e demanda frustrada. Rev Bras Estud Popul 2014; 31:309-31.

23. Gonzaga VAS, Borges ALV, Santos OA, Santa Rosa PLF, Gonçalves RFS. Barreiras organizacionais para disponibilização e inserção do dispositivo intrauterino nos serviços de atenção básica à saúde. Rev Esc Enferm USP 2017; 51:e03270.

24. World Health Organization. Medical eligibility criteria for contraceptive use. https:// apps.who.int/iris/bitstream/handle/ 10665/181468/9789241549158_eng.pdf? sequence $=1$ (acessado em 29/Set/2019).

25. Borges ALV, Santos AO, Araújo KS, Gonçalves RFS, Santa Rosa PLF, Nascimento NC. Satisfação com o uso de métodos contraceptivos entre usuárias de unidades básicas de saúde da cidade de São Paulo. Rev Bras Saúde Matern Infant 2017; 17:757-64. 
26. Murphy PA, Brixner D. Hormonal contraceptive discontinuation patterns according to formulation: investigation of associations in an administrative claims database. Contraception 2008; 77:257-63.

27. Vaughan B, Trussell J, Kost K, Singh S, Jones R. Discontinuation and resumption of contraceptive use: results from the 2002 National Survey of Family Growth. Contraception 2008; 78:271-83.

28. Baumgartner JN, Morroni C, Mlobeli RD, Otterness C, Buga G, Chen M. Impact of a provider job aid intervention on injectable contraceptive continuation in South Africa. Stud Fam Plann 2012; 43:305-14.

29. Chofakian CBN, Moreau C, Borges ALV, Santos OA. Contraceptive discontinuation: frequency and associated factors among undergraduate women in Brazil. Reprod Health 2019; 16:131.
30. Goldstein RL, Upadhyay UD, Raine TR. With pills, patches, rings, and shots: who still uses condoms? A longitudinal cohort study. J Adolesc Health 2013; 52:77-82.

31. Kopp DM, Rosenberg NE, Stuart GS, Miller WC, Hosseinipour MC, Bonongwe P, et al. Patterns of contraceptive adoption, continuation, and switching after delivery among Malawian women. PLoS One 2017; 12:e0170284.

32. Conselho Federal de Enfermagem. Resolução COFEN - 17/2010. Trata-se de encaminhamento dos documentos em epígrafe pela Secretaria do COFEN para análise de Parecer sobre "viabilidade dos enfermeiros realizarem procedimentos com medicamentos e insumos para planejamento familiar reprodutivo. http://www.cofen.gov.br/parecer-n172010-cofen-ctln_6148.html (acessado em 06/Mai/2020). 


\section{Abstract}

The study aimed to estimate the total contraceptive discontinuity rates in the use of oral and injectable hormonal contraceptives, and male condoms and dropout rates due to switches to more effective and less effective methods. Data on 2,051 women, users of primary healthcare services in three Brazilian state capitals, were collected using the contraceptive calendar. The results showed that $24.5 \%$ of users of oral hormonal contraceptives, 33.5\% of users of injectables, and 39\% of users of male condoms had discontinued the respective method after 12 months of use, independently of the reason, and that the rates varied little between the capitals but did depend on the method. The main reason for discontinuing use of the contraceptive method was the desire to become pregnant (20.8\%). Conception while using the method was reported by $20 \%$ of the women, a proportion that reached $25.7 \%$ in users of male condoms. After 12 months with the method, the dropout rate for reasons related to the contraceptive method was $11.4 \%$ in users of injectables; $15.9 \%$ of users of male condoms switched to a more effective method; and $16.3 \%$ of users of injectables switched to a less effective method. Contraceptive discontinuity rates were high and varied according to the contraceptive method.

Contraception; Oral Contraceptives; Hormonal Contraceptive Agents; Condoms; Sexual and Reproductive Health

\section{Resumen}

El objetivo fue estimar las tasas de discontinuidad contraceptiva totales en el uso del contraceptivo hormonal oral o inyectable y del preservativo masculino, así como por abandono, cambio por un método más eficaz o menos eficaz. Se recogieron datos de 2.051 mujeres usuarias de unidades básicas de salud de tres capitales brasileñas mediante el calendario contraceptivo. Los resultados expusieron que un 24,5\% de las usuarias del contraceptivo hormonal oral, un 33,5\% de las usuarias de inyectables y un 39\% de las usuarias del preservativo masculino habian discontinuado el uso del método tras 12 meses de uso, independientemente de la razón, siendo que las tasas poco variaron entre las capitales, pero sí dependiendo del método utilizado. La principal razón para interrumpir el uso del método contraceptivo fue querer quedarse embarazada (20,8\%). Quedarse embarazada mientras se usaba el método fue informado por un 20\% de las mujeres, proporción que alcanza un 25,7\% entre usuarias del preservativo masculino. Se resalta que, tras 12 meses de uso del método, la tasa de abandono por razones relacionadas con el método contraceptivo fue un 11,4\% entre usuarias de los inyectables; la tasa de cambio hacia un método más eficaz fue 15,9\% entre usuarias del preservativo masculino; y la tasa de cambio por un método menos eficaz fue 16,3\% entre usuarias de los inyectables. Las tasas de discontinuidad contraceptiva fueron altas y variaron según el tipo de método contraceptivo utilizado.

Anticoncepción; Anticonceptivos Orales; Agentes Anticonceptivos Hormonales; Condones; Salud Sexual y Reproductiva
Recebido em 27/Jan/2020

Versão final reapresentada em 09/Jul/2020

Aprovado em 11/Jul/2020 